Revue de droit comparé du travail et de la sécurité sociale

3 | 2017

Le travail dans l'économie informelle, un défi pour le droit social

\title{
La Gig Economy
}

Pascale Lorber

\section{OpenEdition}

Journals

Édition électronique

URL : https://journals.openedition.org/rdctss/459

DOI : $10.4000 /$ rdctss.459

ISSN : 2262-9815

Éditeur

Centre de droit comparé du travail et de la sécurité sociale

Édition imprimée

Date de publication : 1 septembre 2017

Pagination : 222-225

ISSN : 2117-4350

\section{Référence électronique}

Pascale Lorber, «La Gig Economy », Revue de droit comparé du travail et de la sécurité sociale [En ligne], 3 | 2017, mis en ligne le 01 novembre 2017, consulté le 04 décembre 2021. URL : http:// journals.openedition.org/rdctss/459; DOI : https://doi.org/10.4000/rdctss.459

\section{(9) $(1) \Theta \Theta$}

Revue de droit comparé du travail et de la sécurité sociale est mise à disposition selon les termes de la Licence Creative Commons Attribution - Pas d'Utilisation Commerciale - Pas de Modification 4.0 International. 


\section{PASCALE LORBER Université de Leicester}

\section{LA GIG ECONOMY}

Comme les chauffeurs d'Amazon, BBC, Amazon Drivers Work Illegal Hours, 11 Novembre 2016, http://www.bbc.co.uk/ news/uk-england-37708996.

Aslam \& Ors $v$ Uber BV \& Ors [2017] IRLR 4 (ET) ; J Prassl, « Who is a worker », Law Quarterly Law Review, 133, 3, 2017, p.366.

Department for Business, Energy and Industrial Strategy, Good Work: the Taylor Review of Modern Working Practices July 2017 https://www.gov.uk/ government/publications/goodwork-the-taylor-review-ofmodern-working-practices.

https://www.thersa.org/ about-us\#.

Qui peut être traduit comme « Travail décent : la revue modernes $\gg\left(n{ }^{\circ} 3\right)$ Taylor des pratiques de travail

Bien qu'il soit encore opportun de faire des commentaires sur le «Brexit », les développements qui suivent porteront sur une question qui a fait l'objet d'une revue significative ces derniers mois : le champ d'application du droit du travail. Les changements que connaissent les modes de travail, notamment ce qu'on appelle au Royaume Uni, la «Gig Economy» sont universels : c'est par exemple, la multiplication des applications permettant au consommateur de réserver directement la prestation et la personne qui l'effectue. Les problèmes soulevés par les modes de fonctionnement de ces nouvelles compagnies (de taxi ou de livraison comme Uber, Deliveroo ou Task Rabbit, qui permet par exemple de demander à une personne de venir faire du repassage ou d'assembler des nouveaux meubles à domicilel sont d'ordre international du point de vue de la révolution numérique. Le Royaume Uni ne fait pas exception.

Le sort des chauffeurs de taxi Uber ou des livreurs Deliveroo ou même des chauffeurs d'Amazon a récemment fait l'objet d'une grande publicité. D'un côté, le journalisme d'investigation nous montre des travailleurs employés par des agences intérimaires, ne bénéficiant d'aucune sécurité de l'emploi, gagnant des sommes en deça du seuil de pauvreté et travaillant durant des horaires illégaux, les « employeurs » se soustrayant également aux normes santé et sécurité ${ }^{1}$. De l'autre, les affaires se multiplient devant les prud'hommes saisis par les chauffeurs Uber ou les livreurs en vélo qui demandent le versement du salaire minimum ou leurs vacances, arguant qu'ils sont des salariés et non des travailleurs indépendants selon ce qu'en a décidé la plateforme pour laquelle ils travaillent ${ }^{2}$.

Ce sont ces abus et ces exploitations qui ont conduit le gouvernement britannique à commander une étude sur les implications des nouvelles formes de travail sur les droits des travailleurs et leurs responsabilités ainsi que sur les libertés et obligations des employeurs ${ }^{3}$. Un portefeuille de six thèmes a été pris en considération : sécurité, salaire et droits ; promotion et formation ; l'équilibre entre droits et responsabilités lincluant le champ d'application du droit du travail pour couvrir les nouvelles formes de travail créées par la « gig economy» et les nouvelles plateformes) ; la représentation des travailleurs; les opportunités pour les groupes sous représentés dans le monde du travail (notamment les personnes souffrant d'un handicap) ; les nouvelles formes de commerce.

La personne chargée de cette étude (Matthew Taylor, un stratège politique également président de la Royal Society of Art, organisme chargé d'enrichir la société par ses idées et ses actions ${ }^{4}$ était assistée de représentants du commerce et d'une juriste de droit du travail. Son rapport a été publié en Juillet 2017 et est intitulé « Good Work : the Taylor Review of Modern Working Practices $»^{5}$. 
Le rapport contient des recommandations de réformes du droit du travail, guidé par sept principes ${ }^{6}$. Ne pouvant les présenter ici en détail, seuls deux aspects essentiels seront analysés. Pour résumer, le but principal du rapport et de ses propositions est de promouvoir le « good work» qui pourrait être traduit par « travail décent » ou travail de qualité à la lecture du rapport. Il est en effet indiqué que tout travail « devrait être juste (ou équitable) et décent $\gg^{7}$. Dans cet esprit, les recommandations du rapport cherchent à s'attaquer à l'exploitation réelle et potentielle au travail, à clarifier le droit applicable et à aider les personnes au travail à connaître et faire appliquer leurs droits ${ }^{8}$. Il est donc

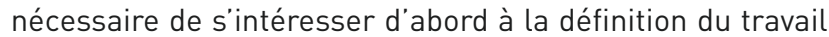
décent et aux raisons pour lesquelles il n'est pas une réalité pour tous (I). Puis, il s'agira de présenter les suggestions faites pour clarifier le champ d'application personnel du droit du travail (II).

\section{Le tRAVAIL dÉCENT ET LE MARCHÉ dU TRAVAIL BRITANNIQUE}

Le rapport commence par tenter de définir ou qualifier le travail décent ${ }^{9}$. Les critères suivants sont utilisés pour mesurer un travail de qualité : le salaire, la qualité de l'emploi (temporaire/ permanent, etc.) ; la formation et les opportunités de développement ; les conditions de travail ; l'équilibre vie personnelle/travail ; la participation et la représentation collective. On indique que les motivations de chaque personne peuvent être différentes vis-à-vis de l'importance accordée à chacun de ces facteurs, la sécurité pouvant être reléguée au second plan si la flexibilité a une valeur plus importante pour le salarié. Cependant, tous ces indicateurs doivent être pris en compte au moment où des réformes sont proposées.

Ces changements s'imposent depuis qu'il y a eu une prise de conscience selon laquelle le marché du travail ne profite pas à tous de la même façon. Les caractéristiques de ce marché ont changé. Bien que le taux de chômage au Royaume-Uni soit un des plus bas en Europe $(4,2 \%)$, on souligne que la productivité et les salaires ne sont pas à un niveau suffisant. Ceci peut être attribué à la qualité des emplois créés. En effet, bien que la majorité des emplois soient encore permanents et à plein temps, plus d'un quart des travailleurs travaillent à temps partiel et le nombre des personnes qualifiées d'indépendants représentent $15 \%$ du marché du travail ${ }^{10}$. De plus, le phénomène du sousemploi lune personne travaille mais le nombre de ses heures n'est pas garanti et elle souhaiterait travailler davantage) est croissant. Enfin, les formes de travail se sont multipliées et ont augmenté : contrats à zéro heure, travail temporaire par exemple. Les statistiques montrent que certains travailleurs apprécient cette flexibilité (volonté de travailler à temps partiel pour un équilibre vie de famille/travail par exemple) mais pour d'autres, cette flexibilité est subie. Ceci peut engendrer des conditions de travail dégradées, des salaires bas, une insécurité d'emploi et une incertitude sur le statut du travailleur. Les affaires devant les tribunaux ou les reportages dans les medias montrent que les employeurs imposent des contrats dans lesquels le travailleur est qualifié de travailleur indépendant alors que les conditions de travail lui sont imposées. Il y a bel et bien subordination 
11 I. Smith, A Baker, O Warnock, Smith and Woods Employment Law, $13^{\text {th }}$ ed, OUP, 2017, p.42.

12 Employment Rights Act 1996, s 230(1).

13 Mummery LJ dans l'affaire Dacas v Brook Street Bureau [2004] IRLR 358 (CA).

14 Taylor Review (n³) p. 35.

Par exemple pour des plombiers dans l'affaire Pimlico Plumbers Ltd v Smith [2017] EWCA Civ 51.

mais l'employeur impose au travailleur un statut de travailleur indépendant - qu'il ne remet pas en cause en raison de sa dépendance économique. C'est ce constat d'exploitation croissante sur le marché du travail que le rapport essaye de mettre en évidence en commençant par un essai de clarification du statut de ces travailleurs.

\section{LE ChAMP d'APPLICATION PERSONNEL DU DROIT dU TRAVAIL}

Le droit du travail britannique est notoirement connu pour son manque de clarté sur le statut des personnes qui travaillent ${ }^{11}$. La législation reconnait les catégories de salariés (A), de travailleur et d'indépendant (B).

A. Le salarié est défini comme une personne ayant un contrat de travail ${ }^{12}$. Ce dernier n'est pas déterminé dans la loi ; c'est la jurisprudence qui a tenté de donner les contours de ce concept. Il en résulte une série de critères appliqués aux situations se présentant devant les juridictions du travail et les tribunaux supérieurs pour déterminer si la personne est salariée ou non. Ceci est fondamental puisque le droit du travail (protection contre licenciement sans cause réelle ou sérieuse ; indemnités pour licenciement économique, etc.) n'est applicable qu'à cette catégorie de personnes. L'incertitude liée à l'application de ces critères a conduit les juges ${ }^{13}$ à en appeler à une intervention parlementaire pour régler la situation notamment des personnes liées par des contrats atypiques (par exemple contrats à zéro heure ou personnes travaillant pour les agences intérimaires), qui sont souvent en situation de vulnérabilité. La revue Taylor propose ainsi que le gouvernement intervienne pour clarifier le statut de salarié par le biais d'une loi. Elle recommande que la loi précise le critère applicable à ce statut, notamment en expliquant les principes à appliquer, laissant aux règlements le soin de fournir plus de détails. Il est d'emblée admis que cette tâche ne sera pas simple ; mais plusieurs témoignages ont souligné cette nécessité de clarification pour assurer une sécurité juridique ${ }^{14}$.

B. Le travailleur est une catégorie qui a été créée dans les années 90 par le gouvernement de Tony Blair pour élargir le champ d'application du droit du travail aux personnes travaillant de façon atypique. L'Employment Right Act 1996, section 230(3) définit le travailleur comme une personne ayant un contrat en vertu duquel elle réalise un travail ou des tâches qu'elle exécute personnellement pour une autre partie dont le statut n'est pas celui de client, de consommateur ou d'entreprise. Ce statut donne accès à un éventail plus limité de droits du travail, par exemple, le salaire minimum ainsi que le droit aux congés payés et à la règlementation sur le temps de travail. Cette catégorie a été récemment largement utilisée par les tribunaux pour requalifier le statut de travailleur indépendant imposé par certaines sociétés aux personnes qu'ils employaient ${ }^{15}$. Bien que cette jurisprudence soit bien reçue par la doctrine et les syndicats, elle n'est pas constante 
car les critères utilisés pour déterminer ce statut ne sont pas toujours clairs ${ }^{16}$. De plus, la jurisprudence reste sujette à changement, notamment en ce qui concerne les décisions récentes impliquant les plateformes telles que Uber qui a clairement indiqué qu'elle ferait appel à l'ensemble des possibilités judiciaires pour faire reconnaitre ses droits ${ }^{17}$.

La revue Taylor a également proposé de reconsidérer le statut de travailleur et de le clarifier, principalement parce qu'il n'est pas toujours facile de distinguer un salarié, un travailleur et une profession libérale. La recommandation est de substituer au terme travailleur celui de " partie contractante dépendante » (dependent contractor). De plus, la définition statutaire devrait également être revue afin de montrer que ces personnes ne sont pas employées mais ne sont pas non plus de réels entrepreneurs. La notion de contrôle devrait constituer l'élément essentiel de cette définition let non celle d'effectuer les tâches pour son propre compte).

Ces mesures restent des recommandations faites au gouvernement. La Première Ministre, Theresa May, a indiqué lors du lancement du rapport qu'elle considèrerait les propositions faites et donnerait un avis d'ici la fin de l'année. Bien que les prémices de départ ne soient pas contestées (la nécessité d'un travail décent), la mise en œuvre a été critiquée. Par exemple, le remplacement de la notion de 'travailleur' par celle de 'contractant dépendant' n'est pas alignée sur la définition de travailleur en droit européen et risque d'accroître le risque de confusion et d'incertitude. La Confédération des Syndicats (TUC) a par exemple argué d'éliminer les trois statuts existants et de ne retenir que deux catégories : travailleur et travailleur indépendant, la première catégorie ayant accès à toute la panoplie des droits du travail, que ces personnes travaillent à zéro heure, pour une agence intérimaire ou une plateforme ${ }^{18}$. La doctrine a également prévenu des dangers de cette approche, notamment la prolifération de ce statut moins protecteur avec les nouvelles formes de travail, laissant ces travailleurs avec moins de protection que les salariés, particulièrement en matière de licenciement ${ }^{19}$. Finalement, au lieu d'utiliser des critères, une solution proposée par les syndicats consisterait à retenir la présomption selon laquelle la personne qui travaille est salariée ${ }^{20}$.

En conclusion, la tentative de réflexion sur les changements du monde du travail, les nouvelles formes d'emploi et comment le droit doit s'y adapter est la bienvenue. Les propositions de réformes incluses dans le rapport de Matthew Taylor sur ce sujet sont clairement bien intentionnées mais ont été critiquées pour ne pas être assez radicales ou précises.
16 J. Prassl $\left(n^{\circ} 2\right)$.

17 The Guardian, « Uber granted right to appeal against ruling on UK drivers' rights », 19 April 2017.

18 H. Reed, «A Third Category is not the right path for Gig Workers: a view from the UK » 6 February 2017, https://onlabor.org/guestpost-a-third-category-is-not-theright-path-for-gig-workers-aview-from-the-uk/ and « 5 reasons why a dependent contractor test is a bad idea », 20 July 2017, http:// touchstoneblog.org.uk/2017/07/5reasons-dependent-contractortest-bad-idea/.

19 The Institute of Employment Rights Guide to the Taylor Review, 13 July 2017, http://www.ier.org. uk/sites/ier.org.uk/files/Taylor $\% 20$ Review\%20recommendations.pdf basé sur un manifeste de réformes du droit du travail rédigé par des universitaires émérites : K. D. Ewing, J. Hendy and C. Jones, $A$ Manisto for Labour Law: towards a comprehensive revision of workers' rights, IER, 2016.

20 TUC, Living on the edge, Summary of the TUC submission to the Taylor Review on the future of work, January 2017, p. 6. 